\title{
Bispectral Analysis of Density and Potential Fluctuations in a High Neutral Density Cylindrical Plasma
}

\author{
Tatsuya KOBAYASHI, Shigeru INAGAKI ${ }^{1)}$, Hiroyuki ARAKAWA, Kunihiro KAMATAKI, \\ Yoshihiko NAGASHIMA ${ }^{2)}$, Takuma YAMADA ${ }^{2)}$, Satoru SUGITA, Masatoshi YAGI ${ }^{1)}$, \\ Naohiro KASUYA ${ }^{3)}$, Akihide FUJISAWA ${ }^{1)}$, Sanae -I. ITOH ${ }^{1)}$ and Kimitaka ITOH $^{3)}$ \\ Interdisciplinary Graduate School of Engineering Sciences, Kyushu University, Fukuoka 816-8580, Japan \\ ${ }^{1)}$ Research Institute for Applied Mechanics, Kyushu University, Fukuoka 816-8580, Japan \\ ${ }^{2)}$ Graduate School of Frontier Sciences, The University of Tokyo, Tokyo 277-8561, Japan \\ ${ }^{3)}$ National Institute for Fusion Science, Toki 509-5292, Japan
}

(Received 7 December 2009 / Accepted 16 March 2010)

\begin{abstract}
The structure of fluctuations in the Large Mirror Device-Upgrade (LMD-U) depends on the neutral density. In this study, we focus on the high neutral density plasma in which the potential and density fluctuation waveforms are nontrigonometric functions similar to sawtooth waves, and peaks in the power spectrum lie along a straight line when plotted as frequency versus wavenumber. Two-dimentional auto and cross bispectral analyses of $\tilde{I}_{\text {is }}$ and/or $\tilde{V}_{\mathrm{f}}$, which consider both the matching conditions for both the azimuthal wavenumber and the frequency, have been performed; significant couplings are observed at particular peak frequencies. Similarities and differences between the auto and cross bicoherence of $\tilde{I}_{\text {is }}$ and/or $\tilde{V}_{\mathrm{f}}$ are discussed.
\end{abstract}

(C) 2010 The Japan Society of Plasma Science and Nuclear Fusion Research

Keywords: linear plasma, drift wave, turbulence, bispectral analysis, nonlinear coupling

DOI: $10.1585 /$ pfr.5.S2047

\section{Introduction}

Recent progress in theoretical and experimental work on plasma turbulence has indicated that mesoscale structures such as zonal flows and streamers are produced by nonlinear mode coupling in drift wave fluctuations. In addition, better understanding of plasma turbulence is considered essential for improving our ability to predict anomalous transport in magnetically confined plasmas. For example, the existence of zonal flows is regarded as crucial to controlling magnetically confined plasmas and realizing a thermonuclear fusion reactor $[1,2]$. Thus, understanding nonlinear mode coupling in drift wave fluctuation is essential [3-8].

Bispectral analysis is useful for investigating nonlinear coupling among three waves that satisfy matching conditions for frequency and/or wavenumber. In a drift wavezonal flow system, energy is exchanged between the zonal flows and the drift wave turbulent fluctuations through three-wave coupling. Thus, coupling between zonal flows and turbulent drift wave fluctuations is observed by applying bispectral analysis [9]. The envelope modulations of turbulent drift wave fluctuations are observed. Moreover, bispectral analysis is used to identify the mutual interaction of drift waves [9-13]. A convergence study of bispectral analysis results [14] has indicated that a large number of repetitions are required to obtain a precise value for the

author's e-mail: kobayashi@riam.kyushu-u.ac.jp bicoherence. Low-temperature fundamental plasmas are very useful because the results of bispectral analysis are highly reproducible. Here, bispectral analysis is applied to low-temperature Large Mirror Device-Upgrace (LMDU) plasma, and many mutual couplings between density fluctuations are identified [13]. In addition to bispectral analysis, cross bispectral analysis between potential and density fluctuations is used to study the coupling between the zonal flows/geodesic acoustic modes (GAMs) and drift wave fluctuations. The cross bicoherence between potential and density fluctuations has not been reported previously for the mutual interactions between drift wave fluctuations. This paper focuses on these mutual interactions. We calculate not only the auto bicoherence of density fluctuations but also the auto bicoherence of potential fluctuations and the cross bicoherence between potential and density fluctuations.

\section{Bispectral Analysis}

The auto bispectrum $\hat{B}$ of the potential fluctuations, which expresses the contribution of the fluctuation energy of three waves to the entire fluctuation energy, is defined as

$$
\hat{B}\left(f_{3}\right)=\left\langle\tilde{\Phi}\left(f_{1}\right) \tilde{\Phi}\left(f_{2}\right) \tilde{\Phi}^{*}\left(f_{3}\right)\right\rangle,
$$

where $f_{3}=f_{1} \pm f_{2}$, and $\tilde{\Phi}(f)$ is the Fourier transformed expression for a temporal waveform of the potential fluctu- 
ation $\tilde{\phi}(t)$. Though we may represent the strength of threewave coupling by the auto bispectrum, it is usually complex and inconvenient to use it to describe the coupling state of the fluctuations. Therefore, instead of the auto bispectrum, we can use the auto bicoherence, which indicates the correlation of the Fourier frequency components between $\tilde{\Phi}\left(f_{1}\right) \tilde{\Phi}\left(f_{2}\right)$ and $\tilde{\Phi}\left(f_{3}\right)$. The auto bicoherence of the potential fluctuations is defined by

$$
\hat{b}^{2}\left(f_{3}\right)=\frac{\left|\hat{B}\left(f_{3}\right)\right|^{2}}{\left\langle\left|\tilde{\Phi}\left(f_{1}\right) \tilde{\Phi}\left(f_{2}\right)\right|^{2}\right\rangle\left\langle\left|\tilde{\Phi}\left(f_{3}\right)\right|^{2}\right\rangle} .
$$

In this paper, the auto bicoherence of the potential and density fluctuations are called the $\phi-\phi-\phi$ coherence and the $n-n-n$ coherence, respectively. Moreover, we can adopt the cross bicoherence by replacing $\tilde{\Phi}\left(f_{3}\right)$ with $\tilde{N}\left(f_{3}\right)$ or $\tilde{\Phi}\left(f_{1}\right) \tilde{\Phi}\left(f_{2}\right)$ with $\tilde{N}\left(f_{1}\right) \tilde{N}\left(f_{2}\right)$ in Eqs. (1) and (2), which express the strength of nonlinear coupling between the potential and density fluctuations, hereafter called the $\phi-\phi-n$ coherence and the $n-n-\phi$ coherence, respectively.

\section{Experimental Setup}

The plasma in the LMD-U is in a cylindrical vacuum vessel with a diameter of about $450 \mathrm{~mm}$ and an axial $(z)$ length of $3740 \mathrm{~mm}$; a schematic is shown in Fig. 1. Plasma is produced using a $3 \mathrm{~kW}, 7 \mathrm{MHz}$ rf source. The magnetic field is $B=0.09 \mathrm{~T}$, The argon pressure in the source region is $p=5$ mTorr, and the electron density and temperature of the LMD-U plasma are typically about $1 \times 10^{19} \mathrm{~m}^{-3}$ and $2-3 \mathrm{eV}$, respectively. In this experiment, relatively high neutral density plasma, in which the collisional drift waves are damped through electron-neutral and ion-neutral collisions, is used, unlike previous work in which relatively low neutral density plasma was used. Temperature fluctuation is assumed to be negligibly small; hence, the fluctuations in floating potential and ion saturation current are considered to be proportional to those of the potential and density, respectively. Fluctuations in the floating potential $\tilde{V}_{\mathrm{f}}$ and the ion saturation current $\tilde{I}_{\text {is }}$ are measured simultaneously with

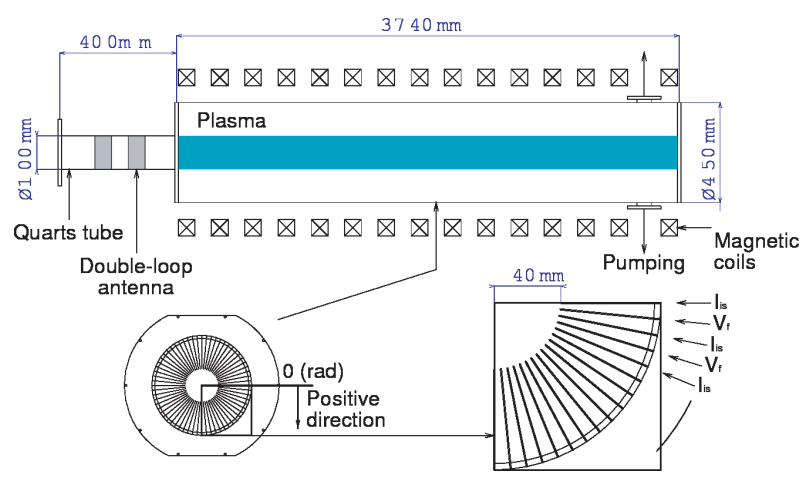

Fig. 1 Schematic of the LMD-U and the 64-channel azimuthal Langmuir probe array. The electrostatic probe's 64 tips are arranged along a $40-\mathrm{mm}$-radius circle. Probe tips measure $\tilde{I}_{\text {is }}$ and $\tilde{V}_{\mathrm{f}}$, alternately. a 64-channel azimuthal Langmuir probe array whose probe tips alternately measure $\tilde{V}_{\mathrm{f}}$ and $\tilde{I}_{\text {is }}$, as shown in Fig. 1 .

\section{Results}

The time evolutions of $\tilde{V}_{\mathrm{f}}$ and $\tilde{I}_{\text {is }}$ are shown in Figs. 2 (a) and (b), respectively. Figure 2 (c) shows the normalized auto power spectra of $e \tilde{V}_{\mathrm{f}} / T_{\mathrm{e}}$ and $\tilde{I}_{\text {is }} / \bar{I}_{\text {is }}$, where $e$ is the elementary charge, and $T_{\mathrm{e}}$ is the electron temperature. Figure 2 (d) shows the ratio of the normalized fluctuation levels. The dashed violet line in $(\mathrm{d})$ indicates a ratio equal to 1 , which is characteristic of drift waves. The crosscoherence and cross-phase between $\tilde{V}_{\mathrm{f}}$ and $\tilde{I}_{\text {is }}$ are calculated at one point in 64 channels, as shown in Fig. 2 (e). $\tilde{V}_{\mathrm{f}}$ and $\tilde{I}_{\text {is }}$ are not measured at same position; hence, the value of $\tilde{V}_{\mathrm{f}}$ at the measuring point for $\tilde{I}_{\text {is }}$ is estimated by averaging the $\tilde{V}_{\mathrm{f}}$ values of two adjoining probes. The twodimensional (2D) (frequency $f$ and poloidal wave number $k_{\theta}$ ) power spectrum of the potential and density fluctuations is also calculated from these spatiotemporal data; its peaks lie along a straight line for frequency versus wave number, like a solitary wave [15].

The spectra of the potential and density fluctuations are characterized by a peak at the fundamental mode $f=$ $1.20 \mathrm{kHz}, m=1$, and its harmonic modes (when the frequency and wave number are nearly proportional, as in a solitary wave). Some side-band modes also appear around each peak. Up to the second harmonic wave, the phase between the potential and density fluctuations is almost zero. On the other hand, the phase of the potential fluctuation is delayed by about $\pi$ from that of the density fluctuation in the modes where $f>3 \mathrm{kHz}$. The magnitude of the normalized auto power spectrum of the density fluctuation is
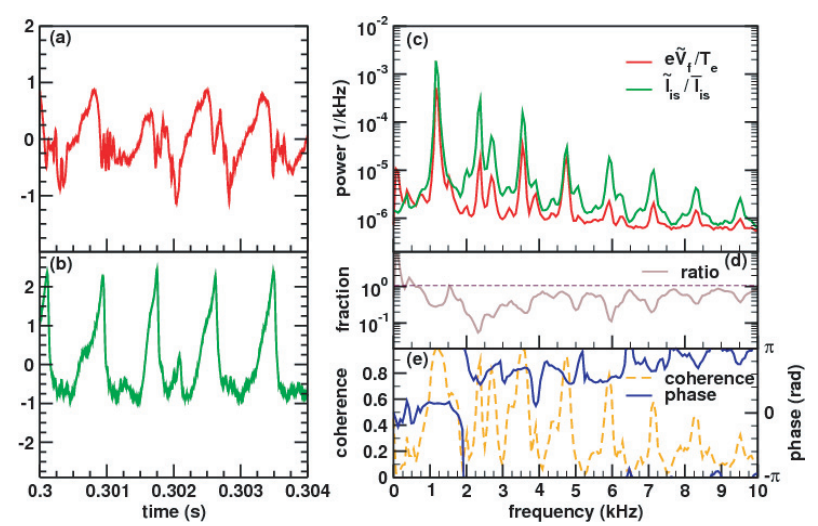

Fig. 2 Information from the 64-channel azimuthal Langmuir probe array. (a), (b) Time evolution of the floating potential $\tilde{V}_{\mathrm{f}}$ and ion saturation current $\tilde{I}_{\text {is }}$, respectively. Their waveforms are similar to sawtooth waves. (c), (d) Normalized auto power spectra of $e \tilde{V}_{\mathrm{f}} / T_{\mathrm{e}}$ and $\tilde{I}_{\mathrm{is}} / \bar{I}_{\mathrm{is}}$ and their ratio, respectively. (e) Cross-coherence and cross-phase of $\tilde{V}_{\mathrm{f}}$ and $\tilde{I}_{\text {is. }}$ A fast Fourier transform is applied with a time-window of $16.38 \mathrm{~ms}$. For (c), 7680 ensembles are averaged and 384 ensembles are averaged for (e). 

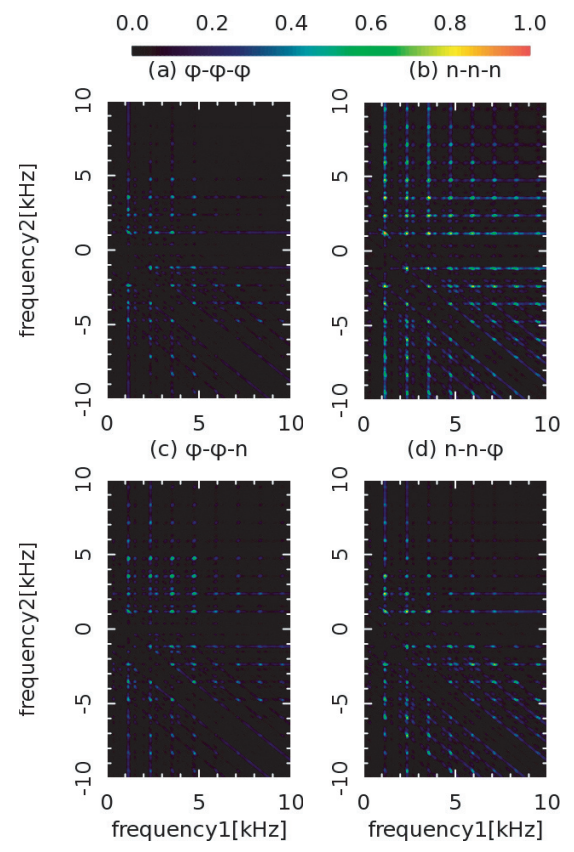

(e)

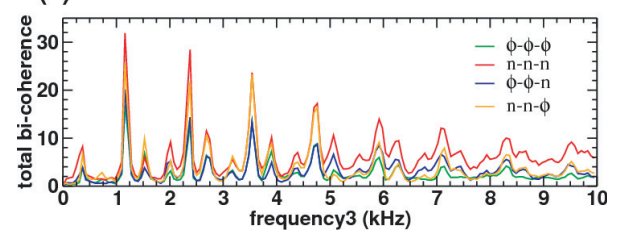

Fig. 3 (a)-(d) Squared auto or cross bicoherence of the $\phi-\phi-\phi$, $n-n-n, \phi-\phi-n$, and $n-n-\phi$ coherence, respectively. (e) Four types of $f_{3}$ dependence of the total bicoherence, which express the strength of nonlinear coupling. Time window is $16.384 \mathrm{~ms}$, and 7680 ensembles are averaged. They are calculated using 20 points of the 64-channel azimuthal Langmuir probe array for ensemble averaging, assuming that their conditions are the same with respect to time.

larger than that of the potential fluctuation in most of the frequency range except for some points. The level of the density fluctuation is of the same order as that of the potential fluctuation at the modes $f=0.793 \mathrm{kHz}, m=1$ and $f=1.53 \mathrm{kHz}, m=1$. In addition, it is smaller at the mode $f=0.366 \mathrm{kHz}, m=0$. The $f=61 \mathrm{~Hz}$ component is regarded as noise from a commercial frequency. As a result, the fundamental mode and its harmonic mode seem to be neither the drift mode nor the flute mode, but another type of instability.

Figures 3 (a)-(d) show the squared auto or cross bicoherence of the $\phi-\phi-\phi, n-n-n, \phi-\phi-n$, and $n-n-\phi$ coherence, respectively. The level of squared bicoherence $\hat{b}^{2}\left(f_{3}\right)$ is indicated by the color bar at the top. The effect of noise on the coherence is suppressed depending on the inverse of the ensemble number $1 / N$ when $N$ is sufficiently large. In this paper, the significance level of bicoherence is estimated to be $\sim 1.3 \times 10^{-3}$. The vertical and horizontal axes indicate $f_{1}$ and $f_{2}$, respectively, and $f_{3}$ is determined by the matching condition $f_{3}=f_{1} \pm f_{2}$. The matching condition $f_{3}=f_{1}-f_{2}$ is called subtractive coupling, and $f_{3}=f_{1}+f_{2}$ is referred to as additive coupling in this paper. Figure 3 (e) shows the $f_{3}$ dependence of the total bicoherence, i.e., the intensity of coupling among the $f_{3}$ mode and all other mode combinations satisfying the matching conditions. Modes that have a high total bicoherence couple nonlinearly with the two other modes that satisfy the matching conditions. According to Fig. 3 (e), significant couplings occur to the $f=0.366 \mathrm{kHz}$ mode and some side-band modes and to the fundamental mode and its harmonics. The total bicoherence at low frequency $\left(f_{3}<5 \mathrm{kHz}\right)$ has remarkably strong peaks. The $\phi-\phi-\phi$ curve is similar to the $\phi-\phi-n$ curve, and the $n-n-n$ curve is similar to the $n-n-\phi$ curve in this frequency range. This tendency is believed to be caused by the high cross-coherence between the potential and density fluctuations at low frequency [Fig. 2(e)]. The total bicoherence at the peak is comparatively suppressed at high frequency $\left(f_{3}>5 \mathrm{kHz}\right)$ relative to that at low frequency. For the case of the $n-n-n$ curve alone, the peaks become broad bands; the similarities at low frequency dissapear.

Figure 4 shows the $f_{1}$ dependence of the bicoherence with respect to a constant $f_{3}$. The value of $f_{2}$ is determined by $f_{2}=f_{3}-f_{1}$ to satisfy the frequency-matching condition. The four curves in Figs. 4 (a) and (b) correspond to Figs. 3 (a)-(d). The width of the fundamental frequency and the $f=0.366 \mathrm{kHz}, m=0$ mode are represented by long and short arrows, respectively. In addition, the violet and brown dashed lines indicate the frequency of the harmonic mode and its side-band mode, respectively. The value of $f_{3}$ we selected is indicated by the blue dashed line. Modes to the left of this line represent a additive coupling, and those to the right represent a subtractive coupling to produce the constant frequency mode we selected. Figure 4 shows (a) the bicoherence for $f_{3}=4.76 \mathrm{kHz}, m_{3}=$ 4 , which is the fourth harmonic wave of the fundamental mode, and (b) that for $f_{3}=2.75 \mathrm{kHz}, m_{3}=2$, which is a side-band mode. Figure 4 (a) indicates that many combinations of two modes couple with mode $f=4.76 \mathrm{kHz}, m=$ 4. For instance, the peak labeled A indicates that modes $f_{1}=3.57 \mathrm{kHz}, m_{1}=3$, and $f_{2}=1.20 \mathrm{kHz}, m_{2}=1$ couple with this mode. One mode at the fundamental frequency or its harmonics couples with the other peaks, indicated by points $\mathrm{A}$ and $\mathrm{B}\left(f_{1}, f_{2}=2.83 \mathrm{kHz}, m_{1}, m_{2}=2\right.$, the second harmonic wave). At $f_{1}=0.793 \mathrm{kHz}$ and $f_{1}=1.53 \mathrm{kHz}$, where the levels of the potential and density fluctuation are same order [Fig. 2 (d)], the levels of the $\phi-\phi-\phi$ and $\phi-\phi-n$ coherence, which are calculated using the potential fluctuation as the $f_{1}$ and $f_{2}$ components, are higher than that of the other two combinations, which use the density fluctuation as the $f_{1}$ and $f_{2}$ components. At other frequencies, the levels of the $\phi-\phi-\phi$ and $\phi-\phi-n$ coherence are lower than that ins the other two combinations. On the other hand, the side-band mode couples with the $f_{1}=0.366 \mathrm{kHz}, m_{1}=0$ mode and a harmonic mode adjoining this side-band mode, shown as point $\mathrm{D}\left(f_{1}=2.38 \mathrm{kHz}, m_{1}=2\right.$, second har- 

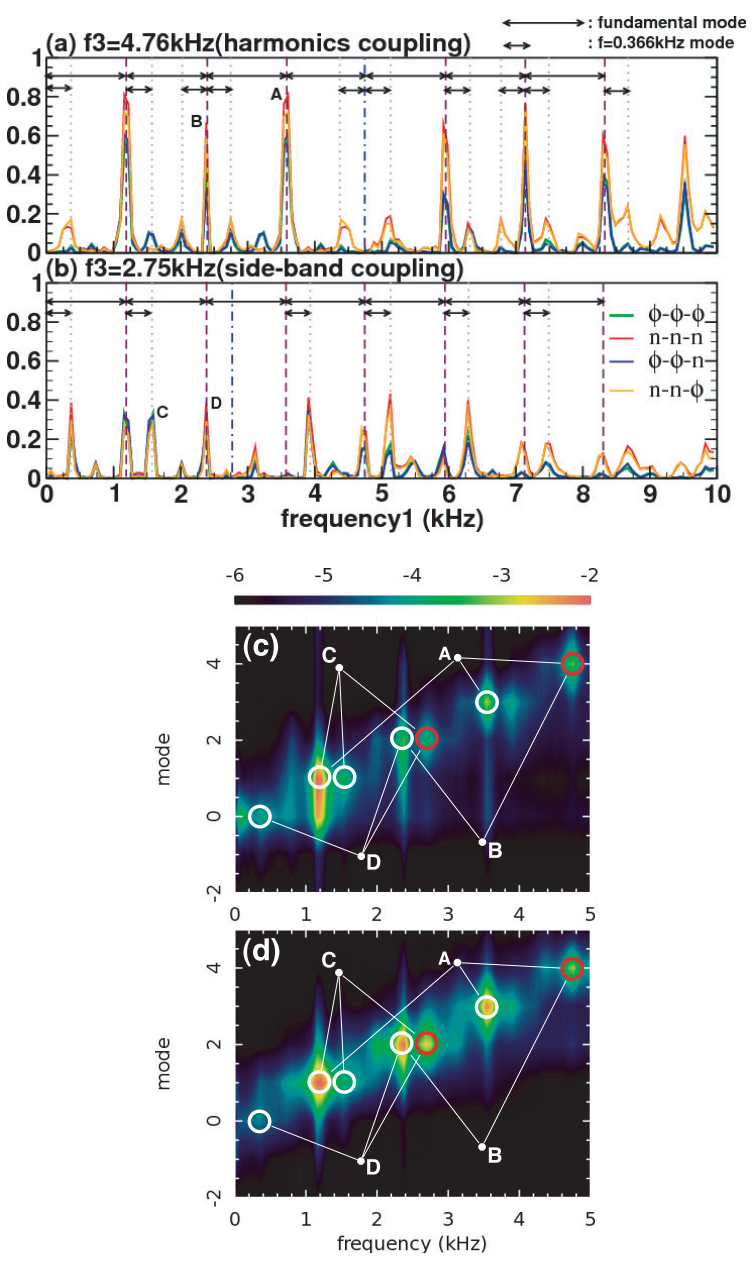

Fig. 4 Squared auto or cross bicoherence with respect to a constant $f_{3}$ are shown. (a) $f_{3}=4.76 \mathrm{kHz}, m_{3}=4$. (b) $f_{3}=$ $2.75 \mathrm{kHz}, m_{3}=2$. Four curves in (a) and (b) correspond to Figs. 3 (a)-(d). (c) 2D spectra of the potential fluctuations; horizontal and vertical axes represent frequency $(\mathrm{kHz})$ and mode number, respectively. (d) 2D spectra of the density fluctuations. Connected circles in (c) and (d) show the significant nonlinear coupling indicated in (a) and (b) by A-D.

monic wave, and $f_{2}=0.366 \mathrm{kHz}, m_{2}=0$ ) in Fig. 4 (b). In addition, coupling with the other side-band mode and harmonic mode is also dominant, as shown by point $\mathrm{C}\left(f_{1}=\right.$ $1.53 \mathrm{kHz}, m_{1}=1$, the side-band mode of the fundamental wave, and $f_{2}=1.20 \mathrm{kHz}, m_{2}=1$, the fundamental wave). The levels of all four curves are similar at low frequency. In contrast, the level of the $n-n-n$ curve is similar to that of the $n-n-\phi$ curve, and that of the $\phi-\phi-\phi$ curve is similar to that of the $\phi-\phi-n$ curve at high frequency. In addition, the $n-n-n$ curve and $n-n-\phi$ curves lie above the $\phi-\phi-\phi$ and $\phi-\phi-n$ curves. These couplings clearly satisfy the matching conditions for not only the frequency, but also the wavenumber, which is shown in Figs. 4 (c) and (d).

\section{Summary}

This study investigated turbulence structures in high neutral density plasma in the LMD-U. In low neutral density plasma in the LMD-U, a streamer has been observed [13]. However, no streamer has been observed in high neutral density plasma. Mutual interaction between drift wave fluctuations is observed in both high and low neutral density plasmas. In addition, the bicoherence of the potential fluctuation and the cross bicoherence between the potential and density fluctuations are observed by simultaneous measurement of the potential and density fluctuations with a 64-channel azimuthal poloidal probe array. The results allow us to discuss the similarities and differences between the auto and cross bicoherence and the density bicoherence. In summary, the bispectral analysis shows that two types of significant nonlinear coupling exist; that related to the fundamental/harmonic modes [Fig. 4 (a)], and that related to the side-band modes and the $f=0.366 \mathrm{kHz}, m=0$ mode [Fig. 4 (b)]. In the former case, although the level of bicoherence of the $\phi-\phi-\phi$ and $\phi-\phi-n$ coherence is higher than that of other two combinations at $f_{1}=0.793 \mathrm{kHz}$ and $f_{1}=1.53 \mathrm{kHz}$, this order is reversed at other frequencies. In the latter case, the level of bicoherence of all four combinations is similar at low frequency $\left(f_{1}<5 \mathrm{kHz}\right)$. In addition, the levels of the $n-n-n$ and $n-n-\phi$ coherence are above those of $\phi-\phi-\phi$ and $\phi-\phi-n$ coherence at high frequency $\left(f_{1}>5 \mathrm{kHz}\right)$. The total bicoherence at low frequency has steep peaks. In contrast, at high frequency, the total bicoherence is relatively smaller. Only the $n-n-n$ coherence indicated the broad band coupling shown to exist at high frequency.

\section{Acknowledgement}

This work was partly supported by a Grant-in-Aid for Scientific Research (S) from the Japan Society for the Promotion of Science (21224014) and the collaboration programs of RIAM at Kyushu University and the National Institute of Fusion Science (NIFS07KOAP017).

[1] P. H. Diamond et al., Plasma Phys. Control. Fusion 47, R35 (2005).

[2] K. Itoh et al., Phys. Plasmas 13, 055502 (2007).

[3] T. Klinger et al., Phys. Rev. Lett. 79, 3913 (1997).

[4] M. Kono et al., Phys. Rev. Lett. 84, 4369 (2000).

[5] C. Schröder et al., Phys. Rev. Lett. 86, 5711 (2001).

[6] G. R. Tynan et al., Plasma Phys. Control. Fusion 48, S51 (2006).

[7] T. Kaneko et al., Phys. Rev. Lett. 90, 125001 (2003).

[8] V. Sokolov et al., Phys. Rev. Lett. 92, 165002 (2004).

[9] Y. Nagashima et al., Plasma Phys. Control. Fusion 48, S1 (2006).

[10] Y. Nagashima et al., Phys. Rev. Lett. 95, 9, 90905 (1-8) (2005).

[11] Y. Nagashima et al., Plasma Phys. Control. Fusion 49, 1611 (2007).

[12] T. Yamada et al., Plasma Fusion Res. 3, S1021 (2008).

[13] T. Yamada et al., Nature Phys 4, 721 (2008).

[14] D. Gresillon et al., Phys. Fluids 31, 1904 (1988).

[15] H. Arakawa et al., Plasma Phys. Control. Fusion 51, 85001 (2009). 